\title{
Thermal plasmonic interconnects in graphene
}

\author{
Baoan Liu, ${ }^{1}$ Yongmin Liu, ${ }^{2}$ and Sheng Shen ${ }^{1, *}$ \\ ${ }^{1}$ Department of Mechanical Engineering, Carnegie Mellon University, Pittsburgh, Pennsylvania 15213, USA \\ ${ }^{2}$ Department of Mechanical \& Industrial Engineering, Department of Electrical \& Computer Engineering, \\ Northeastern University, Boston, Massachusetts 02115, USA
}

(Received 12 March 2014; revised manuscript received 7 October 2014; published 10 November 2014)

\begin{abstract}
As one emerging plasmonic material, graphene can support surface plasmons at infrared and terahertz frequencies with unprecedented properties due to the strong interactions between graphene and low-frequency photons. Since graphene surface plasmons exist in the infrared and terahertz regime, they can be thermally pumped (excited) by the infrared evanescent waves emitted from an object. Here we show that thermal graphene plasmons can be efficiently excited and have monochromatic and tunable spectra, thus paving a way to harness thermal energy for graphene plasmonic devices. We further demonstrate that "thermal information communication" via graphene surface plasmons can be potentially realized by effectively harnessing thermal energy from various heat sources, e.g., the waste heat dissipated from nanoelectronic devices. These findings open up an avenue of thermal plasmonics based on graphene for different applications ranging from infrared emission control, to information processing and communication, to energy harvesting.
\end{abstract}

DOI: 10.1103/PhysRevB.90.195411

PACS number(s): 81.05.ue, 44.40.+a, 73.20.Mf

\section{INTRODUCTION}

The manipulation of optical fields and energy flow of light plays a pivotal role in communication and information technologies. Among various means of controlling light, surface plasmons (SPs), known as the coupled excitations of photons and free electrons, enable confinement and control of electromagnetic energy at the subwavelength scale and thus bridge optics and nanotechnology. Noble metals such as gold and silver are widely used plasmonic materials in the visible and near-infrared range. However, metal SPs in the infrared and terahertz range (e.g., dual-conductor transmission-line modes [1]) usually have a large wavelength comparable to that of free-space photons and thus cannot truly squeeze the light down to deep subwavelength scale, especially in propagating direction. Recent studies have shown that graphene, a two-dimensional single layer of carbon atoms, can support propagating SPs with unprecedented properties at infrared and terahertz frequencies due to the strong interactions between graphene and low-frequency photons [2,3]. More importantly, graphene surface plasmons (GSPs) can be confined to an extremely small dimension, on the order of $10^{2}$ times smaller than the diffraction limit, and can be tuned over a wide range by gating or doping [3]. Hence, graphene has emerged as a promising plasmonic material for tunable infrared or terahertz light sources [4], subwavelength optical circuits [5], robust and cost-effective photodetectors for terahertz radiation [6-11], and nanoelectronic devices with strong light-matter interactions [12], to name a few.

In spite of the unique properties of GSPs, launching GSPs remains challenging because of the large wave-vector mismatch between GSPs and free-space light waves [13]. Moreover, active light sources currently used for exciting GSPs, such as infrared lasers, cannot be easily miniaturized and integrated into optoelectronic circuits [2,14]. Since GSPs exist in the infrared and terahertz regime, they can be thermally

*Corresponding author: sshen1@ @ cmu.edu excited by the infrared evanescent waves emitted from an object [15]. However, compared with active light sources like lasers, thermal emission usually has a broad spectrum with low output power limited by the blackbody radiation [16]. In this paper, by directly solving stochastic Maxwell's equations, we show that thermal GSPs can be efficiently excited and have monochromatic and tunable spectra. Furthermore, we demonstrate that thermal energy can be employed to transmit information via GSPs, enabling information communication with negligible cross talk. These results provide understanding of graphene in the areas of thermal science and nano-optics, implying that we can harness thermal energy to build various graphene plasmonic devices.

\section{THERMAL EXCITATION OF MONOCHROMATIC AND TUNABLE GSPS}

The key for efficiently launching monochromatic GSPs is to utilize the resonance of nanoscale thermal emitters. Previously, graphene nanoribbons and nanodisks have been discovered as high- $Q$ plasmonic cavities, whose resonance frequencies can be tuned by designing their Fermi level and geometry [3]. Here we employ graphene nanoribbon thermal emitters (surface plasmon cavities in two dimensions) to excite GSPs on a coupled graphene sheet. To directly simulate the thermal radiation energy transfer and field intensity of graphene, we solve stochastic Maxwell's equations by both the fluctuating-surface-current (FSC) formulation and the Wienerchaos-expansion (WCE) formulation under the framework of the two-dimensional (2D) boundary element method (BEM) $[17,18]$ (see Appendix). As shown in the schematic of Fig. 1(a), a graphene nanoribbon thermal source with width $W$ and Fermi level $E_{F}$ is placed in proximity to a graphene sheet at a nanoscale gap $d$. Similar suspended graphene nanoribbon structures have been experimentally realized in Refs. [19-21]. Electromagnetic energy output from a thermal source is usually understood as the radiation from thermally induced random dipoles in the source [22]. When the nanoribbon emitter is close to graphene, the evanescent photons with 
(a)

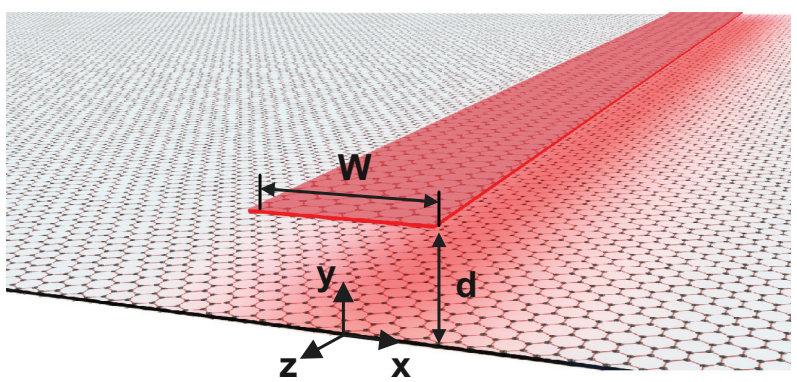

(b)

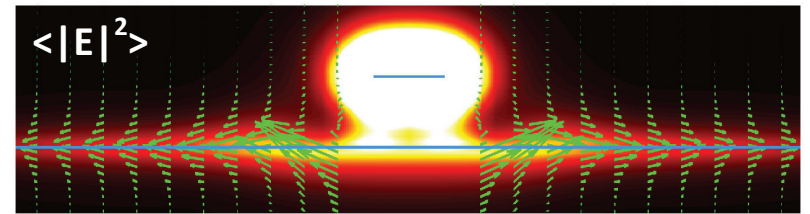

(c)

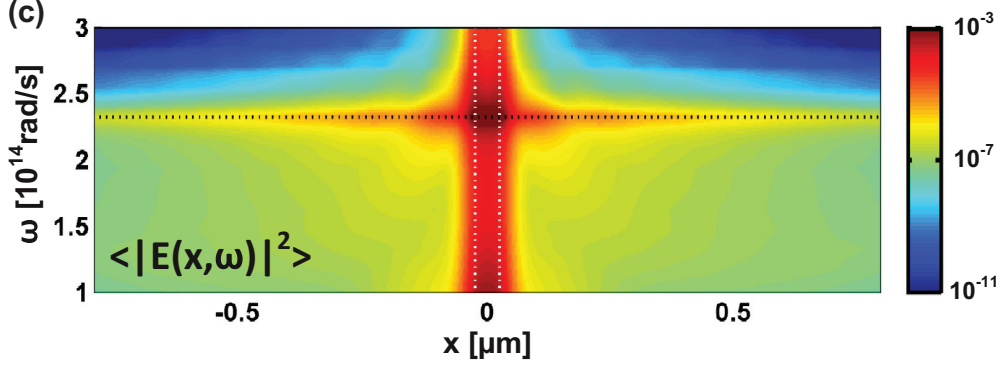

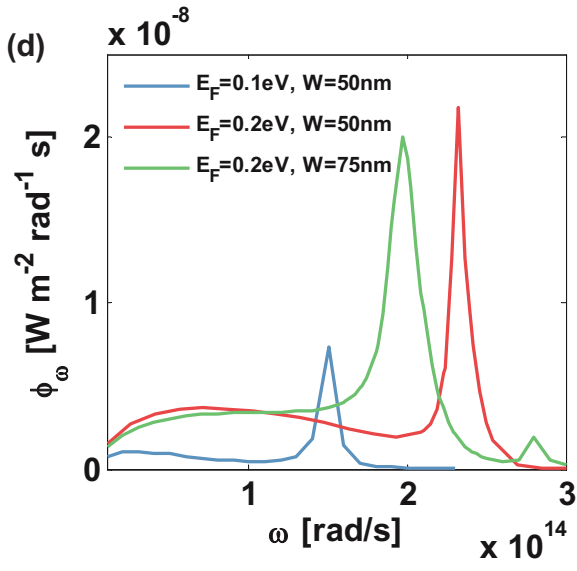

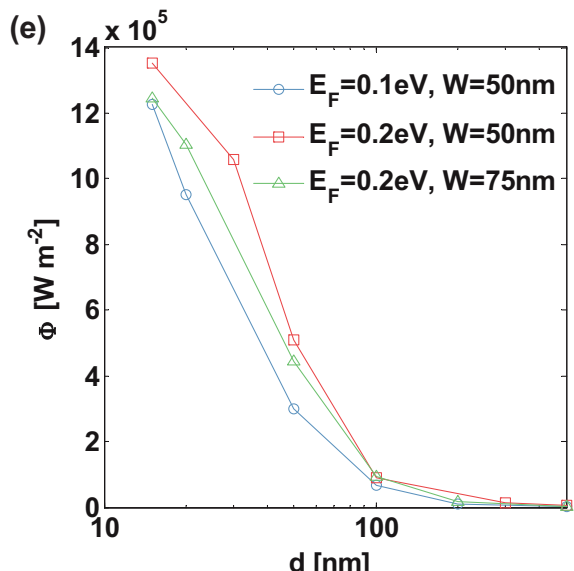

FIG. 1. (Color online) (a) Schematic of a graphene nanoribbon thermal emitter and a graphene sheet. (b) Electric field intensity $\left\langle|E|^{2}\right\rangle$ (color plot) and Poynting vector (green quiver plot) due to the thermal emission from the nanoribbon emitter. Blue lines indicate the locations of the graphene ribbon and sheet. (c) Spectral field intensity $\left\langle|E(x, \omega)|^{2}\right\rangle$ (in the unit of $\mathrm{V}^{2} \mathrm{~m}^{-2} \mathrm{rad}^{-1} \mathrm{~s}$ and plot in log scale) along the $x$ direction and at $15 \mathrm{~nm}$ above the graphene sheet. The white dashed lines indicate the location of the graphene nanoribbon emitter. The black dashed line indicates the peak frequency $\left(\omega=2.3 \times 10^{14} \mathrm{rad} / \mathrm{s}\right)$ of the thermally excited GSPs. (d) Spectral energy fluxes of the thermally excited GSPs by graphene nanoirbbon emitters with different $W$ and $E_{F}$, at the same gap $d=50 \mathrm{~nm}$. (e) The total power $\Phi$ (normalized to the area of nanoribbon) of the thermally excited GSPs as a function of $d$.

large wave vector produced by the random dipoles enable the excitation of surface waves on the graphene sheet. In addition, thermal emission from a heat source in the near field (at a nanoscale gap) is not limited by the blackbody radiation [23].

For the case of $d=50 \mathrm{~nm}, W=50 \mathrm{~nm}$, and $E_{F}=0.2 \mathrm{eV}$, the thermal radiation field intensity and energy flow profiles (cross-sectional view) due to the graphene nanoribbon emitter are illustrated in Fig. 1(b). Here the nanoribbon thermal emitter and the graphene sheet are maintained at 500 and $0 \mathrm{~K}$, respectively, in order to demonstrate the effect of the nanoribbon thermal source. The mobility of graphene is set to be $10000 \mathrm{~cm}^{2} \mathrm{~V}^{-1} \mathrm{~s}^{-1}$, which can be achieved by exfoliated graphene samples. The field intensity profile in Fig. 1(b) shows that the thermal radiation of the nanoribbon emitter excites the confined surface waves on the graphene sheet, which can spread a long distance from the emitter. The Poynting vector profile [green arrows in Fig. 1(b)] further confirms that the surface waves indeed carry energy and propagate away from the nanoribbon emitter. Due to the near-field effect, the output energy flux from the nanoribbon emitter to the sheet is $7 \times 10^{5} \mathrm{~W} / \mathrm{m}^{2}$ (total power normalized to the ribbon area), which is 2 orders of magnitude larger than the blackbody limit. Moreover, the graphene nanoribbon thermal emitter is essentially an extremely efficient source for exciting graphene surface waves because of the significant enhancement of the photon local density of states (PLDOS) in the near field [24]. In this case, almost $100 \%$ of the energy output from the nanoribbon is converted to surface waves through evanescent photons, and only $0.01 \%$ of the energy output leaks to the far-field surroundings due to propagating photons.

To investigate the spectrum of the thermally excited surface waves, the spectral field intensity along the graphene sheet is plotted in Fig. 1(c). It can be clearly seen that only the field intensity at $\omega=2.3 \times 10^{14} \mathrm{rad} / \mathrm{s}$ spreads a long distance in the $x$ direction on the graphene sheet [highlighted by the black dashed line in Fig. 1(c)], which indicates the monochromatic excitation of the propagating GSPs. By designing the cavity modes (e.g., tuning the Fermi level and ribbon size), the peak frequency of the thermally excited GSPs by the graphene nanoribbon can also be controlled, as shown in Fig. 1(d). For example, the $Q$ factor is estimated to be $\sim 20$ for the graphene ribbon cavity with $E_{F}=0.2 \mathrm{eV}$ and $W=50 \mathrm{~nm}$ [the red 
curve in Fig. 1(d)]. Despite the monochromatism, the thermally excited GSPs are incoherent because the source dipoles inside the emitter are random. Furthermore, the power of the thermally excited GSPs significantly depends on the ribbon-sheet separation due to the near-field effect. As shown in Fig. 1(e) for graphene ribbons with different sizes and Fermi levels, the total power dramatically increases with reduced gap size.

To understand the mechanism of the thermal excitation of monochromatic GSPs, we perform the mode expansion of the thermally emitted photons in the $\left(k_{z}, \omega\right)$ space. There are two fundamental reasons to perform the mode expansion in the $\left(k_{z}, \omega\right)$ space: (1) Since the complex graphene structures proposed in this paper are 2D invariant (in the $z$ direction), only the $z$ component of wave vector $k_{z}$ and frequency $\omega$ of each photon are conserved. (2) The 2D BEM (together with the FSC formulation and the WCE method) allows us to calculate the exact contribution from each photo mode $\left(k_{z}, \omega\right)$ to the energy flux and the field intensity.

The total surface waves excited on the graphene sheet in Fig. 1(b) include the contributions from "long-range" propagating surface waves and "quickly decaying" surface waves. For the excited GSPs on the graphene sheet in Fig. 1(a) (except the projection area of the nanoribbon), its surface wave vector, $k_{s}=\sqrt{k_{x}^{2}+k_{z}^{2}}$, should be equal to $k_{\mathrm{GSP}}=\frac{\omega}{c_{0}} \sqrt{1-\frac{4 \epsilon_{0}}{\mu_{0} \sigma_{g}^{2}(\omega, T)}}$, the surface wave vector of intrinsic GSPs on a single graphene sheet. Its $k_{x}$ needs to be a real number in order to "propagate" or carry energy in the $x$ direction. Therefore we divide the mode space $\left(k_{z}, \omega\right)$ into two regions by the dispersion curve $k_{\mathrm{GSP}}(\omega)$ of the intrinsic GSPs on a graphene sheet, as shown by the white curves in Fig. 2(a). The first region corresponds to the modes with $k_{z}<k_{\mathrm{GSP}}$, which are capable of exciting the propagating surface waves with real $k_{x}$. The second region represents the modes with $k_{z}>k_{\mathrm{GSP}}$, which can only excite the quickly decaying surface waves due to the imaginary $k_{x}$. To further clarify the physical meaning of the two-mode regions in Fig. 2(a), we plot the thermal radiation field profiles $\left\langle|E|^{2}\right\rangle$ due to the modes from these two regions, as shown in Fig. 2(c). It can be clearly seen that only the first mode region $\left(k_{z}<k_{\mathrm{GSP}}\right)$ contributes to the excitation of the propagating GSPs, while the second mode region $\left(k_{z}>k_{\mathrm{GSP}}\right)$ only leads to the quickly decaying surface waves, which are highly localized near the nanoribbon and do not carry substantial energy in the $x$ direction.

As a result, the upper limit of the output power exciting propagating GSPs can be calculated by integrating the photon energy flux due to the first mode region $\left(k_{z}<k_{\mathrm{GSP}}\right)$. The corresponding upper bound of the excitation efficiency of propagating GSPs is estimated to be $70 \%$ for the case with $d=50 \mathrm{~nm}$, where the excitation efficiency is defined as the ratio between the energy flux carried by propagating GSPs and the total energy output from the nanoribbon emitter. Here, we also want to emphasize that the exact excitation efficiency of propagating GSPs depends on both the graphene material property and the structure geometry in real cases.

The separation between the graphene nanoribbon emitter and the graphene sheet can also strongly influence the excitation of GSPs. In order to excite GSPs, a small gap is required because only the evanescent photons can match the large wave vector of GSPs. However, if the gap is too small, the monochromatism of excited GSPs vanishes due to (a)

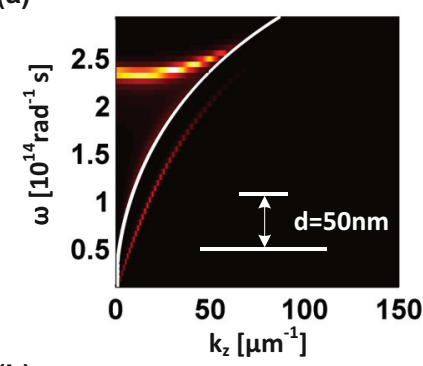

(c)

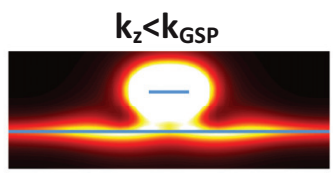

(b)
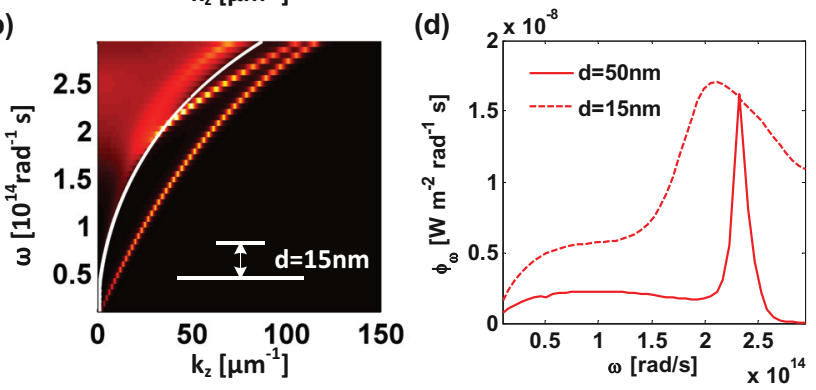

FIG. 2. (Color online) (a) and (b) Contribution from each photon mode $\left(k_{z}, \omega\right)$ to the thermal excitation of GSPs on the graphene sheet for (a) $d=50 \mathrm{~nm}$ and (b) $d=15 \mathrm{~nm}$, respectively. The graphene nanoribbon emitter has $E_{F}=0.2 \mathrm{eV}$ and $W=50 \mathrm{~nm}$. The white curves represent the dispersion relation of intrinsic GSPs on a single graphene sheet. (c) Thermal radiation field profile due to the photon modes from the two regions: $k_{z}<k_{\mathrm{GSP}}$ and $k_{z}>k_{\mathrm{GSP}}$. (d) The spectral energy flux carried by the surface waves with $k_{z}<k_{\mathrm{GSP}}$ for both $d=50 \mathrm{~nm}$ and $d=15 \mathrm{~nm}$ cases.

the strong coupling between the nanoribbon and the sheet. In Fig. 2(d), we plot the spectral energy flux carried by the surface waves with $k_{z}<k_{\mathrm{GSP}}$ for both $d=50 \mathrm{~nm}$ and $d=15 \mathrm{~nm}$. The frequency band of GSPs for $d=15 \mathrm{~nm}$ becomes much broader compared with the $d=50 \mathrm{~nm}$ case. The mechanism can also be revealed by the results of mode expansion. For the $d=50 \mathrm{~nm}$ case, the monochromatism of the thermally excited GSPs is attributed to the narrow bright area in the first mode region $\left(k_{z}<k_{\mathrm{GSP}}\right)$ in Fig. 2(a), which corresponds to the first-order cavity mode (or edge mode) of an individual graphene nanoribbon due to the optical confinement in the $x$ direction. This consistence of the resonance modes in a graphene ribbon-sheet system with an individual graphene ribbon indicates the weak coupling between the ribbon and the sheet. For the $d=15 \mathrm{~nm}$ case, the resonance modes of the graphene nanoribbon are quite different from those of the 50-nm gap due to the strong coupling between the ribbon and the sheet. As shown in Fig. 2(b), there exist no single "resonance modes" in the first mode region $\left(k_{z}<k_{\mathrm{GSP}}\right)$, which contribute to the excitation of propagating surface waves.

\section{INFORMATION COMMUNICATION VIA THERMAL GSPS}

Based on the properties of the GSPs that are thermally excited by a graphene nanoribbon emitter, we design and demonstrate "thermal GSP interconnects." Superior to conventional optical circuits, GSP interconnects can achieve the thinnest possible platform and field confinement on the order 
of 10-100 nm, 100 times smaller than the infrared wavelength. In comparison with conventional SP interconnects, GSP interconnects work at the infrared range, and they can be easily tuned by modifying the Fermi level of graphene. To prove the concept, we study the systems which are composed of graphene nanoribbon transmitters and receivers. All the nanoribbons are separated from the graphene waveguide with a proper gap size so that they are all weakly coupled to the waveguide. A graphene nanoribbon transmitter transmits signals by being heated to a high temperature (e.g., by the Joule heating from an electrically biased graphene nanoribbon) and then exciting the GSPs thermally. Likewise, the graphene nanoribbon transmitter can be electrically modulated at high frequency, considering the extremely small thermal mass of the graphene nanoribbon. A graphene nanoribbon receiver receives or detects signals by absorbing the energy carried by GSPs. To date, graphene-based infrared light detectors have been demonstrated [6-11], all of which are required to absorb the light directly by graphene. Furthermore, due to the resonance property of graphene nanoribbons, the proposed thermal GSP interconnect allows us to selectively transmit information at the desired spatial and spectral positions.

The first system illustrated in Fig. 3(a) is composed of one transmitter $\mathrm{A}$ and two receivers $\mathrm{B}$ and $\mathrm{C}$, all of which are 50-nm-wide nanoribbons and are separated from the graphene sheet waveguide with a 50-nm gap. To demonstrate the idea of long-range communications, the distances between $\mathrm{A}$ to $\mathrm{B}$ and $\mathrm{B}$ to $\mathrm{C}$ are set at $1.5 \mu \mathrm{m}$, which is long enough to prevent the direct near-field coupling between adjacent nanoribbons. The Fermi level and mobility of the graphene sheet waveguide are set to be $E_{F}=0.6 \mathrm{eV}$ and $\mu=10000 \mathrm{~cm}^{2} \mathrm{~V}^{-1} \mathrm{~s}^{-1}$, respectively. In this case, the propagation distance of GSPs reaches $10 \mu \mathrm{m}$. Transmitter $\mathrm{A}$ is at $500 \mathrm{~K}$, and the rest of the system, including the graphene waveguide and receivers $\mathrm{B}$ and $\mathrm{C}$, is maintained at $300 \mathrm{~K}$. Although the heated graphene waveguide can also excite GSPs thermally, they have no
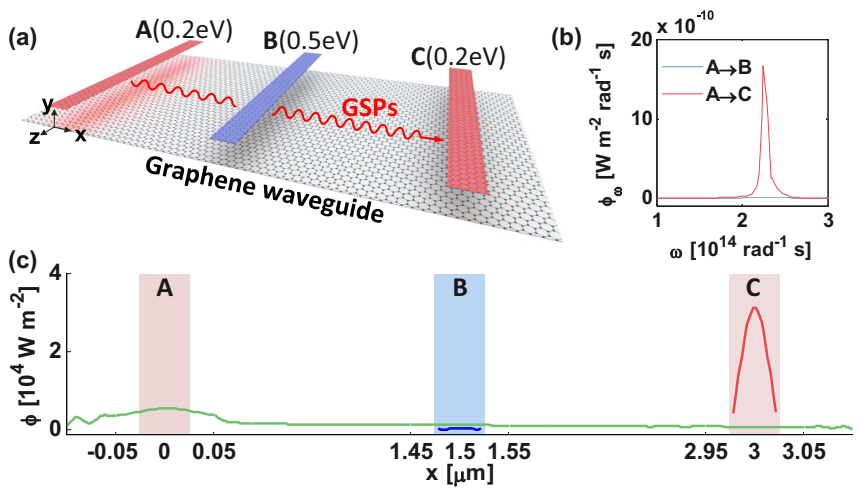

FIG. 3. (Color online) (a) Schematic of a basic demonstration in which graphene nanoribbon $\mathrm{A}$ is a transmitter and nanoribbons $\mathrm{B}, \mathrm{C}$ are receivers. The graphene sheet with a finite width of $4 \mu \mathrm{m}$ serves as a GSP waveguide. The gap between the nanoribbons and the sheet is $50 \mathrm{~nm}$. (b) Spectral energy fluxes for $\mathrm{A} \rightarrow \mathrm{B}$ (blue curve) and $\mathrm{A} \rightarrow \mathrm{C}$ (red curve). The results are normalized to the area of the nanoribbon transmitter. (c) Local energy flux profiles on receiver B (blue curve), receiver $\mathrm{C}$ (red curve), and graphene waveguide (green curve). The red and blue regions indicate the locations of the graphene ribbons. impact on receivers because there is no net energy flow from the waveguide to receivers. The information cannot be transferred without net energy flow in a reciprocal thermal system [25]. Transmitter $A$ and receiver $C$ have the same resonance frequency because both the Fermi levels are equal to $0.2 \mathrm{eV}$, whereas receiver $\mathrm{B}$ is designed to have a different resonance frequency by setting its Fermi level to be $0.5 \mathrm{eV}$. Practically, the Fermi level of graphene can be tuned by chemical doping or electric gating [26]. The energy flux spectra between transmitter $\mathrm{A}$ and receivers $\mathrm{B}$ and $\mathrm{C}$ are plotted in Fig. 3(b). Although receiver B is closer to transmitter A than receiver $C$, receiver $C$ receives much more energy than receiver $\mathrm{B}$ due to the match of graphene ribbon resonance frequency. The weak coupling between nanoribbons ensures that the information communication between transmitter $\mathrm{A}$ and receiver $\mathrm{C}$ is not interfered by receiver $\mathrm{B}$. The weak coupling also leads to the high efficiency of the waveguide. The local energy flux distribution at the peak frequency of energy flux received by receiver $C\left(\omega=2.3 \times 10^{14} \mathrm{rad} / \mathrm{s}\right)$ is shown in Fig. 3(c). The energy dissipated in the graphene waveguide underneath receiver $C$ (green curve) is negligible compared to the energy absorbed by receiver $\mathrm{C}$ (red curve). The total thermal energy fluxes from transmitter A to receivers $\mathrm{B}$ and $\mathrm{C}$ are calculated to be $3.7 \times 10^{2}$ and $1.8 \times 10^{4} \mathrm{~W} / \mathrm{m}^{2}$, respectively, where the results are normalized to the area of the receiver. The high energy flux results from the near-field effect between the graphene ribbon and sheet, which has been demonstrated to be several orders of magnitude larger than the blackbody radiation. Quantitatively, the energy flux from transmitter A to receiver $C$ is negligibly small $\left(3.1 \mathrm{~W} / \mathrm{m}^{2}\right)$ if the graphene sheet waveguide is absent. The dominant energy dissipation mechanism in this system is the intrinsic loss of GSPs on the graphene waveguide, which can be reduced by using high-mobility graphene.

The monochromatism of thermally excited GSPs allows the multichannel thermal information communication on a single waveguide, providing the possibility of integrating multiplegraphene-based thermoplasmonic devices together to form a complex GSP interconnection system. Consider a system with two transmitters $\mathrm{A}$ and $\mathrm{B}$ and two receivers $\mathrm{C}$ and $\mathrm{D}$, as shown in Fig. 4(a). The Fermi levels of receivers $A$ and $C$ are set to be $0.2 \mathrm{eV}$, whereas the Fermi levels of receivers $\mathrm{B}$ and $\mathrm{D}$ are set to be $0.1 \mathrm{eV}$. The width of the nanoribbons, the gap between the nanoribbons and the graphene waveguide, and the material properties of the graphene waveguide are chosen to be the same as with the previous system in Fig. 3. The graphene nanoribbons are also separated from their adjacent nanoribbon by a large distance of $1.5 \mu \mathrm{m}$. The energy flux spectra of this system are plotted in Fig. 4(b). Since thermally excited GSPs by transmitters A and B are monochromatic at different frequencies, the cross-talk energy transfer of $\mathrm{A} \rightarrow \mathrm{D}$ or $\mathrm{B} \rightarrow \mathrm{C}$ is about 1 order of magnitude smaller than the energy transfer through the desired channels $(\mathrm{A} \rightarrow \mathrm{C}$ or $\mathrm{B} \rightarrow$ D), as shown in Fig. 4(b).

\section{CONCLUSION}

In summary, thermal excitation of GSPs provides unprecedented opportunities for both thermal science and nanophotonics. Our numerical calculations demonstrate that 


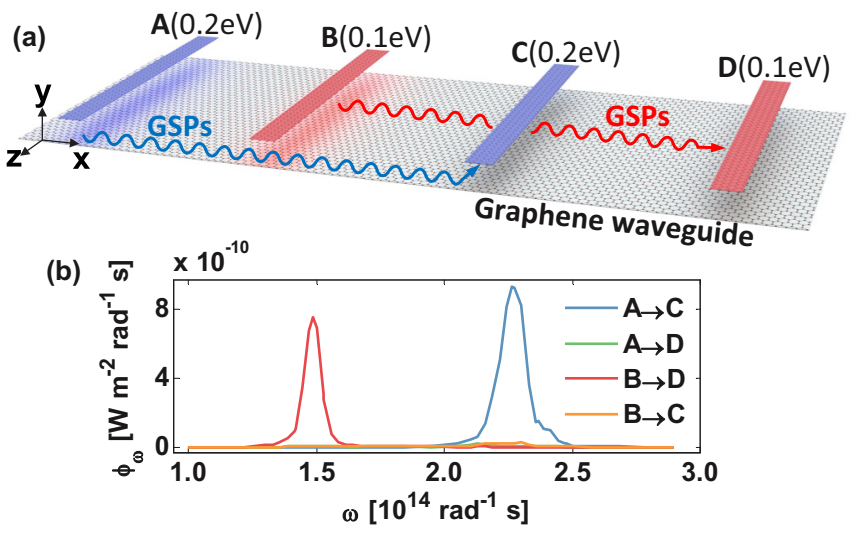

FIG. 4. (Color online) (a) Schematic of two graphene nanoribbon transmitters and two nanoribbon receivers. The graphene sheet waveguide has the finite width of $6.4 \mu \mathrm{m}$. (b) Spectral energy transfer for different transmitter and receiver pairs. The total energy fluxes for $\mathrm{A} \rightarrow \mathrm{C}$ and $\mathrm{B} \rightarrow \mathrm{D}$ are $1.3 \times 10^{4}$ and $6.6 \times 10^{3} \mathrm{~W} / \mathrm{m}^{2}$, respectively. The "cross-talk" energy fluxes for A $\rightarrow \mathrm{D}$ and B $\rightarrow \mathrm{C}$ are $4.4 \times 10^{2}$ and $7.6 \times 10^{2} \mathrm{~W} / \mathrm{m}^{2}$, respectively. The results are normalized to the area of the corresponding nanoribbon transmitter.

thermal energy can be efficiently converted into the monochromatic and tunable propagating GSPs. Therefore, the plasmonics theory and methods can be applied to manipulate the thermal energy (e.g., GSP waveguide, GSP modulator, GSP detector, etc.). Graphene nanoemitters can be designed to have specific output spectra as guided by the optical cavity theory. More importantly, the power of thermally excited GSPs is not limited by the blackbody radiation due to near-field energy transfer. While the exfoliated graphene samples with high mobility demonstrate the potential to be infrared SP waveguides, other low-loss infrared SP waveguides (e.g., dual-conductor transmission line metal waveguide) can also be adopted to transmit thermal signals in this scenario. This is because the near-field waves radiated by the graphene nanoemitter can also excite the propagating SP transmissionline modes. As a result, "thermal GSP interconnects" can be realized to effectively harness thermal energy and used to transmit information.

\section{ACKNOWLEDGMENT}

The authors acknowledge funding support from National Science Foundation (CBET-1253692).

\section{APPENDIX A: THERMAL RADIATION SIMULATION}

The principle of directly calculating thermal radiation is to exactly solve stochastic Maxwell's equations. Under local thermal equilibrium, the thermally induced random currents $j$ are incoherent and their intensities can be evaluated by the fluctuation dissipation theorem: $j(r, \omega)=V(\omega, T) d W(r)$, where $V(\omega, T)=\sqrt{\frac{4}{\pi} \epsilon_{0} \operatorname{Im}\left[\epsilon_{r}\right] \frac{\hbar \omega}{\exp \left[\hbar \omega / k_{B} T\right]-1}}$ is a deterministic function related to the relative permittivity $\epsilon_{r}$ of the material, the local temperature $T$, and frequency $\omega$, and $d W(r)$ is the white noise function indicating the spatial incoherence. After incorporating the random current $j$ into Maxwell's equations, thermal radiation can be calculated by averaging the energy flux or the energy density of electromagnetic waves from the random currents. In addition, using stochastic electrodynamics to calculate the thermal radiation of graphene and other materials has been justified by previous experimental works [27,28]. To solve stochastic Maxwell's equations, rather than evaluating the contribution from each incoherent random dipole inside emitters, which is extremely computationally expensive, we apply the FSC formulation [17] and the WCE formulation [18] to evaluate the thermal radiation energy flux and the field profile of graphene.

\section{Fluctuating-Surface-Current formulation}

Under the framework of the BEM, the FSC formulation calculates the thermal radiation energy flux with $O\left(N^{3}\right)$ computation steps, where $N$ is the number of boundary elements used to discretize the geometries. It is as efficient as solving deterministic Maxwell's equations with coherent sources in the BEM (e.g., calculating energy absorption of objects due to an external plane wave). Specifically, in local thermal equilibrium, the FSC formulation states that the ensemble-averaged flux spectrum

$$
\begin{aligned}
\phi(\omega)= & \frac{2}{\pi} \operatorname{Tr}\left[\operatorname{sym}\left(G_{e}\right)\left[M^{-1}\right]^{*} \operatorname{sym}\left(G_{a}\right) M^{-1}\right] \\
& \times\left[\Theta\left(\omega, T_{1}\right)-\Theta\left(\omega, T_{2}\right)\right],
\end{aligned}
$$

where $\quad \operatorname{sym}(X)=\frac{1}{2}\left[X+X^{*}\right], \quad \Theta(\omega, T)=\hbar \omega /[\exp (\hbar \omega /$ $\left.k_{B} T\right)-1$ ] is the Planck distribution. As specified in Ref. [17], $G_{e}$ (or $G_{a}$ ) is the self-interaction matrix that describes the electromagnetic interactions between any two boundary elements on the surface of a thermal emitter (or absorber). $M$ is the BEM matrix and is equal to the summation of all the self-interaction matrices of each body (the exterior vacuum space is also counted as a body). The local energy flux can be obtained from the main diagonal terms of the resultant matrix inside the bracket of Eq. (A1).

\section{Wiener-chaos expansion formulation}

The WCE formulation not only calculates the thermal radiation energy flux, but also provides the field intensity profile. The underlying principle is to apply the Wiener chaos expansion to expand the random currents $j$ using the deterministic orthonormal basis functions $\left\{f_{i}(r)\right\}$ defined inside the thermal emitters, i.e.,

$$
j(r, \omega)=V(\omega, T) d W(r)=V(\omega, T) \sum_{i} c_{i} f_{i}(r),
$$

where $\left\{c_{i}\right\}$ are the independent random variables [18]. As a result, the thermal radiation energy flux and field intensity due to the random currents are equal to the summation of the contributions from each deterministic current distribution $j_{i}=V(\omega, T) \times f_{i}(r)$. Furthermore, if the orthonormal basis is chosen in the multipole expansion form (e.g., the sinusoidal basis for a graphene nanoribbon), Eq. (A2) can be fast converging. Therefore the summation can be truncated, and a certain number of lower-order terms are enough to achieve good accuracy. The WCE formulation can also be highly efficient with the computational time complexity of $O\left(N^{3} M\right)$ under the framework of BEM, where $M$ is the number of basis 
functions being expanded. In our case, by choosing $\left\{f_{i}(r)\right\}$ in the sinusoidal forms, the result from the WCE formulation with the first six modes $(M=6)$ agrees well with the one from the FSC formulation.

\section{APPENDIX B: RESISTIVE BOUNDARY MODEL OF GRAPHENE}

Instead of approximating the atomic-thin graphene as a finite-thickness dielectric thin film with permittivity $\epsilon=$ $1+i \sigma_{G} /\left(\epsilon_{0} \omega t\right)$ ( $t$ is the thickness), we model the graphene as a zero-thickness resistive boundary with a surface conductivity of $\sigma_{G}$, where $\sigma_{G}$ is calculated based on the equations in Ref. [29]. In comparison with the thin-film approximation, modeling graphene as a resistive boundary in the BEM is more accurate, efficient, and numerically stable. The validity of the resistive boundary in the BEM is described in Ref. [30]. Furthermore, we employ the principle of graphene thermal radiation based on the fluctuating electrodynamics formulation in Ref. [31]. The selfinteraction matrix of graphene in the BEM can be expressed as $G_{m n}^{\text {graphene }}=\left\langle\beta_{m}, \frac{1}{\sigma_{G}} \beta_{n}\right\rangle$.
[1] M. Schnell, P. Alonso-González, L. Arzubiaga, F. Casanova, L. E. Hueso, A. Chuvilin, and R. Hillenbrand, Nat. Photonics 5, 283 (2011)

[2] J. Chen, M. Badioli, P. Alonso-González, S. Thongrattanasiri, F. Huth, J. Osmond, M. Spasenović, A. Centeno, A. Pesquera, P. Godignon, A. Z. Elorza, N. Camara, F. J. García de Abajo, R. Hillenbrand, and F. H. L. Koppens, Nature (London) 487, 77 (2012).

[3] F. H. L. Koppens, D. E. Chang, and F. J. García de Abajo, Nano Lett. 11, 3370 (2011).

[4] I. Gierz, J. C. Petersen, M. Mitrano, C. Cacho, I. C. E. Turcu, E. Springate, A. Stöhr, A. Köhler, U. Starke, and A. Cavalleri, Nat. Mater. 12, 1119 (2013).

[5] Y.-M. Lin, A. Valdes-Garcia, S.-J. Han, D. B. Farmer, I. Meric, Y. Sun, Y. Wu, C. Dimitrakopoulos, A. Grill, P. Avouris, and K. A. Jenkins, Science 332, 1294 (2011).

[6] M. Freitag, T. Low, W. Zhu, H. Yan, F. Xia, and P. Avouris, Nat. Commun. 4, 1951 (2013).

[7] J. Yan, M.-H. Kim, J. A. Elle, A. B. Sushkov, G. S. Jenkins, H. M. Milchberg, M. S. Fuhrer, and H. D. Drew, Nat. Nanotechnol. 7, 472 (2012).

[8] N. Nader Esfahani, R. E. Peale, C. J. Fredricksen, J. W. Cleary, J. Hendrickson, W. R. Buchwald, B. D. Dawson, and M. Ishigami, Proceedings SPIE 8261, Terahertz Technology and Applications V, San Francisco, CA (SPIE, Bellingham, WA, 2012), p. 82610E.

[9] M. Furchi, A. Urich, A. Pospischil, G. Lilley, K. Unterrainer, H. Detz, P. Klang, A. M. Andrews, W. Schrenk, G. Strasser, and T. Mueller, Nano Lett. 12, 2773 (2012).

[10] F. Xia, T. Mueller, Y.-M. Lin, A. Valdes-Garcia, and P. Avouris, Nat. Nanotechnol. 4, 839 (2009).

[11] T. Mueller, F. Xia, and P. Avouris, Nat. Photonics 4, 297 (2010).

[12] M. Liu, X. Yin, E. Ulin-Avila, B. Geng, T. Zentgraf, L. Ju, F. Wang, and X. Zhang, Nature (London) 474, 64 (2011).

[13] A. N. Grigorenko, M. Polini, and K. S. Novoselov, Nat. Photonics 6, 749 (2012).
[14] W. Gao, G. Shi, Z. Jin, J. Shu, Q. Zhang, R. Vajtai, P. M. Ajayan, J. Kono, and Q. Xu, Nano Lett. 13, 3698 (2013).

[15] O. Ilic, M. Jablan, J. D. Joannopoulos, I. Celanovic, H. Buljan, and M. Soljačić, Phys. Rev. B 85, 155422 (2012).

[16] R. Siegel, Thermal Radiation Heat Transfer, 4th ed. (Taylor \& Francis, UK, 2001).

[17] A. W. Rodriguez, M. T. H. Reid, and S. G. Johnson, Phys. Rev. B 88, 054305 (2013).

[18] B. Liu and S. Shen, Phys. Rev. B 87, 115403 (2013).

[19] S. Yiğen and A. R. Champagne, Nano Lett. 14, 289 (2014).

[20] C. Chen, S. Rosenblatt, K. I. Bolotin, W. Kalb, P. Kim, I. Kymissis, H. L. Stormer, T. F. Heinz, and J. Hone, Nat. Nanotechnol. 4, 861 (2009).

[21] I. Neumann, J. Van de Vondel, G. Bridoux, M. V Costache, F. Alzina, C. M. Sotomayor Torres, and S. O. Valenzuela, Small 9, 156 (2013).

[22] S. M. Rytov, Y. A. Kravtsov, and V. I. Tatarskii, Principles of Statistical Radiophysics, Vol. 3 (Springer-Verlag, Berlin, 1989).

[23] K. Joulain, J.-P. Mulet, F. Marquier, R. Carminati, and J.-J. Greffet, Surf. Sci. Rep. 57, 59 (2005).

[24] J. B. Pendry, J. Phys.: Condens. Matter 11, 6621 (1999).

[25] J. B. Pendry, J. Phys. A: Math. Gen. 16, 2161 (1983).

[26] M. F. Craciun, S. Russo, M. Yamamoto, and S. Tarucha, Nano Today 6, 42 (2011).

[27] P. J. van Zwol, S. Thiele, C. Berger, W. A. de Heer, and J. Chevrier, Phys. Rev. Lett. 109, 264301 (2012).

[28] S. Shen, A. Narayanaswamy, and G. Chen, Nano Lett. 9, 2909 (2009).

[29] L. A. Falkovsky, J. Phys.: Conf. Ser. 129, 012004 (2008).

[30] L. N. Medgyesi-Mitschang, J. M. Putnam, and M. B. Gedera, J. Opt. Soc. Am. A 11, 1383 (1994).

[31] O. Ilic, M. Jablan, J. D. Joannopoulos, I. Celanovic, and M. Soljačić, Opt. Express 20, A366 (2012). 